\title{
Estudio demoscópico sobre los atributos de un dispositivo de asistencia abdomino-intestinal.
}

\author{
Esnal-Angulo, Iñaki ${ }^{a}$ \& Hernandis-Ortuño, Bernabéb \\ ${ }^{a} \mathrm{PhD}$ candidate - Universitat Politècnica de València, Spain - iesnalangulo@gmail.com \\ ${ }^{\mathrm{b}}$ Full Professor - Universitat Politècnica de València, Spain - bhernand@upv.es
}

\section{Resumen}

Se presenta el estudio de la opinión de posibles usuarios y/o consumidores realizado sobre los atributos de diseño de un asistente abdominointestinal para ayudar a paliar el estreñimiento. El objetivo del estudio es la identificación de las características más relevantes que debe poseer un producto de dicha tipología y la detección de los grupos poblacionales más interesantes mediante el análisis y la valoración de la opinión de los posibles usuarios. El estudio llevado a cabo fue de carácter cuantitativo y consistió en una encuesta autocumplimentada realizada a 411 individuos, 225 mujeres (54.7\%) y 186 varones (45.3\%); seleccionados mediante muestreo aleatorio y realizados cara a cara en centros sanitarios y a través de redes sociales y mensajería electrónica. El cuestionario constó de 40 preguntas de valoración tipo Likert sobre el nivel de acuerdo o desacuerdo de los ítems propuestos y 6 preguntas de perfil. Los resultados obtenidos destacan la identificación de varios grupos poblacionales con intereses muy dispares, como son las mujeres y hombres de mediana edad, quienes valoran positivamente la funcionalidad y negativamente el consumo eléctrico y la necesidad de aprendizaje de uso, respectivamente. Por otro lado, los grupos de personas mayores de 60 años, con escaso nivel de estudios muestran una preocupación por el uso supervisado por los especialistas en centros sanitarios; y los menores de 30 años con alto nivel de estudios, quienes comparten su descontento con el uso en centros especializados, pero valoran positivamente que sea ecológico, personalizable y robusto. Se concluye que la diferenciación de estos grupos obliga a la consideración de diferentes propuestas de diseño en función de los intereses de los distintos perfiles detectados con el fin de adecuar el producto al mayor número de consumidores potenciales.

Palabras clave: Diseño, atributos, producto, estreñimiento, usuarios. 


\begin{abstract}
The study of the opinion of possible users and / or consumers on the design attributes of an abdominointestinal assistant to help alleviate constipation is presented. The aim of the study is the identification of the most relevant characteristics that such typology of a product must possess and the detection of the most interesting population groups by means of the analysis and the evaluation of the opinion of the possible users. The observational study consisted of a self-completed survey of 411 individuals, 225 women (54.7\%) and 186 men (45.3\%); selected through random sampling and performed face to face in health centers and through social networks and electronic messaging. The questionnaire consisted of 40 Likert-type assessment questions on the level of agreement or disagreement of the proposed items and 6 profile questions considering age, gender, nationality, level of education, constipation, and evacuation frequency. The results obtained highlight the identification of several population groups with very different interests, such as middle-aged women and men, who value above all the functionality and negatively the electric consumption and the need for learning of use, respectively. On the other hand, groups of people over 60 years of age, with low level of studies show a concern about the use supervised by specialists in health centers; and those under 30 with a high level of education, who share their discontent with the use in specialized centers, but value positively that it is ecological, customizable and robust. It is concluded that the differentiation of these groups forces the consideration of different design proposals according to the interests of the different profiles detected in order to adapt the product to the greater number of potential consumers.
\end{abstract}

Keywords: Design, Attributes, Product, Constipation, Users.

\title{
1. Introducción
}

A pesar de la existencia de un creciente interés por el estudio de los factores de diseño centrados en el usuario (UCD), resulta todavía difícil determinar cuáles son los aspectos mejor valorados por los consumidores en lo concerniente a productos cuya tipología aún no ha sido contemplada por el mercado. Muchos de los estudios realizados en este sentido se basan en la experiencia de las propias empresas en función de las ventas o lo resultados 
estadísticos obtenidos de las estrategias de marketing (Norman, 1988). Cada vez más, se contemplan otras herramientas basadas en el "Big data" como fuente de información para determinar cuáles son los posibles consumidores o los más propensos a adquirir nuevos productos que aún no han salido al mercado (López García, 2013). Esta práctica conlleva gran cantidad de información y equipos de analistas especializados, así como una gran cantidad de recursos que, en muchas ocasiones, pequeñas o medianas empresas no pueden asumir.

Es el caso por ejemplo de la temática propuesta, un dispositivo mecánico-funcional para reducir el estreñimiento idiopático mediante el abordaje postural y dinámico (Esnal \& Serrano, 2013). La peculiar e inédita perspectiva sobre la que se aborda la temática propuesta y el carácter íntimo de su naturaleza, conlleva que la opinión de los posibles usuarios y/o consumidores se presente de crucial importancia para el resultado final del producto terminado y la afinidad que éste pueda tener con la persona que lo va a comprar y, especialmente, a utilizar. Los factores sociales, culturales, económicos, políticos, étnicos, etc... influyen directamente en los requerimientos de diseño y preestablecen los condicionantes para el desarrollo del producto, que en ocasiones y como es el caso, se trata de una temática delicada y tabú aún en muchas regiones.

Como consecuencia de ello, los factores correspondientes a la ergonomía emocional, a priori menos evidentes, emergen de la interacción generada entre el usuario y el producto resultando fundamentales en la psicología del usuario hacia el producto como la percepción de la calidad, la empatía, y por tanto la aceptación del producto por el usuario y/o consumidor.

Es por esta razón que se recurre habitualmente a la utilización de estudios demoscópicos para la obtención de información sobre las características que deben poseer los futuros productos de uso humano, garantizando un cierto nivel de afinidad con los futuros usuarios y/o consumidores (Malhorta, 2004).

Se presenta un caso de estudio en el que se pretende una solución mecánica de diseño para los posibles usuarios, no necesariamente pacientes diagnosticados, que necesitan de una paliación de la sintomatología del estreñimiento funcional idiopático. Según considera la Organización Mundial de Gastroenterología (WGO, 2010) el abanico de posibles pacientes es muy amplio y aunque se pueden establecer grupos de mayor riesgo, sostienen que dos tercios de la población mundial occidental lo han sufrido o lo sufrirán por lo menos una vez a lo largo de su vida.

Esta perspectiva tan amplia obliga a cercar el estudio y centrarlo en grupos poblacionales más reducidos con el fin de establecer con el mayor nivel de rigurosidad posible las características adecuadas para un producto de semejantes pretensiones como el considerado. 


\section{Metodología}

Se llevó a cabo el estudio demoscópico como herramienta para la identificación de los aspectos más relevantes para el abordaje del diseño de un dispositivo como el propuesto desde la perspectiva del usuario, así como para la categorización de los diferentes grupos de consumidores potenciales.

El estudio consistió en una encuesta orientada a posibles usuarios y consumidores en la que se consideró una muestra poblacional representativa de la población española de aproximadamente 46 millones de personas (INE, 2016). La muestra fue calculada según la siguiente ecuación de intervalo de confianza (Arriaza, 2005):

$$
n=\frac{N * \sigma^{2} * Z^{2}}{(N-1) e^{2}+\sigma^{2} * Z^{2}}
$$

Dónde:

$$
\begin{aligned}
& \mathrm{n}=\text { Muestra poblacional; } \\
& \mathrm{N}=\text { Población española; } \\
& \sigma=\text { Desviación estándar; } \\
& \mathrm{Z}=\text { Nivel de confianza; } \\
& \mathrm{e}=\text { Error muestral; }
\end{aligned}
$$

Los valores utilizados para el cálculo de la muestra necesaria para representar a la población total española se establecieron de la siguiente manera: una desviación estándar del $50 \%(\sigma=0.5)$, un nivel de confianza del 95\% (1.96) y un error muestral del $5 \%(\mathrm{e}=$ $0.05)$.

La encuesta fue realizada tanto cara a cara con el encuestado, como electrónicamente. Una parte de la encuesta $(118,28.9 \%)$ fue realizada a pie de calle a las puertas del Hospital Clínico Universitario de Valencia, el Hospital "La Fe", el Hospital "Nisa Valencia al mar" el Centro de Salud de Benimaclet, el campus de la Universitat Politécnica de València, el campus de la Universidad de Valencia y aledaños. La otra parte de la encuesta $(289,71 \%$ aprox.) fue realizada telemáticamente y divulgada a través de diferentes plataformas sociales, pudiendo ser ésta una condición de sesgo a tener en cuenta, a priori, en la interpretación de los resultados, tanto por la edad del usuario como por las diferencias entre los diversos medios de divulgación. 
El estudio está formado, en primer lugar, por una introducción en la que se plantea el proyecto de investigación y el motivo de la encuesta. Tras la presentación se le muestran varias imágenes de productos de naturaleza semejante para ayudar a visualizar y entender mejor la cuestión planteada. En segundo lugar, consta de un formulario de 2 únicas preguntas desglosadas en apartados. La primera consta de 40 apartados y se refiere a los atributos que debe poseer un producto basado en el dispositivo abdominointestinal. Está estructurado en base a la escala de valoración Likert de 0 a 3, en función del nivel de acuerdo o desacuerdo del propio usuario, donde $0=$ Totalmente en desacuerdo, $1=$ En desacuerdo, 2 = De acuerdo y $3=$ Totalmente de acuerdo. La segunda pregunta, consta de 6 apartados y se refiere a las cuestiones acerca del perfil de usuario. Se consideraron los criterios de género, edad, nivel de estudios, si padece estreñimiento y la frecuencia de evacuación para la caracterización de la muestra. El software de análisis estadístico utilizado fue el SPSS (v11).

La categorización de la muestra se llevó a cabo mediante el cruce y comparación de los factores de la muestra con las tablas de contingencia para medir la dependencia entre dichos factores y su nivel de significación. Se realizó una selección de dichas dependencias centrando el interés en las que mostraron un nivel de significación asintótica de chicuadrado de Pearson (p-value) por debajo de 0.05. Las que por el contrario mostraban un valor superior fueron descartadas.

El análisis descriptivo de la información recogida fue realizado mediante la aplicación del modelo ANOVA basado en la comparación de las medias de las variables independientes con respecto a las variables dependientes de la muestra. De la misma manera se centró el estudio en las relaciones cuya dependencia entre variables es verdaderamente significativa y, por consiguiente, útil. Por tanto, se realizó de nuevo una selección de los factores que mostraban un valor de significación por encima de 0.95 (p-value $<0.05$ ). De igual manera, los que presentaban valores inferiores fueron descartados. Los resultados obtenidos se recogieron en tablas y se representaron gráficamente para facilitar la interpretación y comprensión de los datos.

\section{Resultados}

Los análisis observacionales y descriptivos de la muestra arrojaron, en primera instancia, que la distribución presenta una mayor participación de las mujeres $(225,54.7 \%)$ frente a los hombres (186, 45.3\%). Los rangos de edades más participativos fueron los de 30 a 39 años $(115,28 \%$ ), seguidos de los grupos de madurez (de 40 a 49 años $(84,20.4 \%$ ), de 50 a 59 años $(78,19 \%)$ y de 60 a 69 años $(74,18 \%))$. Los extremos, menores de 20 años (8, $1.9 \%$ ) y mayores de 69 años $(5,1.2 \%)$ de la muestra son los que menor participación presentan. El criterio Nivel de estudios, destaca al grupo Universitarios (157, 38.2\%) como 
el de mayor participación, seguido de los de Primaria $(90,21.9 \%)$ y bastante equidad en los siguientes grupos de Secundaria $(68,16.5 \%)$ y Bachiller $(75,18.2 \%)$. La población muestral consideró que sufrir estreñimiento es un fenómeno mayoritariamente ocasional $(263,64 \%)$, mientras que no sufrirlo nunca $(59,14.4 \%)$ y frecuentemente $(74,18 \%)$ obtienen valores cercanos. Un $3.6 \%$ de la muestra (15) reconocen sufrirlo permanentemente. Sin embargo, la frecuencia de evacuación que más prevalece es la de 1 ó 2 veces al día $(224,54.4 \%)$, esto significa que por regla general la muestra se puede considerar regular. No obstante cabe destacar el $22.4 \%$ de la muestra (92) está en el límite, según la definición de Roma III (Rome Fundation, 2006) y el 10.7 \% (44) que corresponde a personas estreñidas.

Tabla 1. Tabla de contingencia Género * Nivel de estudios.

\begin{tabular}{|c|c|c|c|c|c|c|c|}
\hline \multirow[b]{2}{*}{ Género } & \multicolumn{5}{|c|}{ Nivel de estudios } & \multirow[b]{2}{*}{ Total } & \multirow[b]{2}{*}{ P-value } \\
\hline & $\begin{array}{c}\text { Sin } \\
\text { estudios }\end{array}$ & Primaria & ESO & Bachiller & Universitario & & \\
\hline Hombre & $11(5.9 \%)$ & $26(14 \%)$ & $31(16.7 \%)$ & $50(26.9 \%)$ & $68(36.6 \%)$ & 186 & \\
\hline Mujer & $10(4.4 \%)$ & $64(28.4 \%)$ & $37(16.4 \%)$ & $25(11.1 \%)$ & $129(39.6 \%)$ & 225 & 0.000 \\
\hline Total & $21(5.1 \%)$ & $90(21.1 \%)$ & $68(16.5 \%)$ & $75(18.2 \%)$ & $154(38.2 \%)$ & 411 & \\
\hline
\end{tabular}

En la relación que presenta el Género con respecto al Nivel de estudios, tal y como se puede observar en la tabla anterior (Tabla 1), se destaca a las mujeres por encima de los hombres en Primaria $(64,28.4 \%)$ y estudios universitarios $(129,57.3 \%)$, mientras que los valores para los hombres son más elevados en Bachiller (50, 26.8\%). Una posible lectura de los datos es que se combinan dos grupos, uno de mayor edad, procedentes de generaciones en las que la mujer ha tenido mayores dificultades para acceder a la formación; y otro de menor edad en donde las mujeres poseen un rol de mayor participación.

Tabla 2. Tabla de contingencia Género * Sufre estreñimiento.

\begin{tabular}{|c|c|c|c|c|c|c|}
\hline \multirow{2}{*}{ Género } & \multicolumn{4}{|c|}{ Sufre estreñimiento } & \multirow{2}{*}{ Total } & \multirow{2}{*}{$\begin{array}{c}\text { P- } \\
\text { value }\end{array}$} \\
\hline & Nunca & Ocasional & Frecuente & Permanente & & \\
\hline Hombre & 32 & 114 & 40 & 0 & 186 & \\
\hline Mujer & 27 & 149 & 34 & 15 & 225 & 0,001 \\
\hline Total & 59 & 263 & 74 & 15 & 411 & \\
\hline
\end{tabular}


En cuanto a sufrir estreñimiento, se destaca que las mujeres muestran una exclusividad total en el grupo de Permanente. De lo que se puede extraer que el sector femenino es más propenso al estreñimiento crónico, corroborando así, la información recogida de las fuentes consultadas en alusión a considerar a este grupo como el más sensible o de mayor riesgo de padecerlo (AEG, 2011).

Tabla 3. Tabla de contingencia Género * Frecuencia de evacuación.

\begin{tabular}{|c|c|c|c|c|c|c|c|}
\hline \multirow[b]{2}{*}{ Género } & \multicolumn{5}{|c|}{ Frecuencia de ir al baño } & \multirow[b]{2}{*}{ Total } & \multirow{2}{*}{$\begin{array}{c}\text { P- } \\
\text { value }\end{array}$} \\
\hline & $\begin{array}{c}1-2 \\
/ \mathrm{s}\end{array}$ & $\begin{array}{c}3-4- \\
/ s\end{array}$ & $\begin{array}{c}5-6 \\
/ s\end{array}$ & $\begin{array}{c}1-2 \\
/ d\end{array}$ & $\begin{array}{l}30 \text { más } \\
/ \text { d }\end{array}$ & & \\
\hline Hombre & 20 & 49 & 15 & 99 & 3 & 186 & \\
\hline Mujer & 24 & 43 & 33 & 125 & 0 & 225 & 0.042 \\
\hline Total & 44 & 92 & 48 & 224 & 3 & 411 & \\
\hline
\end{tabular}

A pesar de la superioridad numérica de las mujeres en la muestra, los valores de los hombres se muestran semejantes para los niveles más altos de estreñimiento, lo que podría corresponder con la frecuencia de padecerlo anteriormente mencionada. También es notable la contundencia de la frecuencia de mayor rango (3 o más deposiciones al día) por parte de este grupo.

Tabla 4. Tabla de contingencia Edad * Sufre estreñimiento.

\begin{tabular}{|c|c|c|c|c|c|c|}
\hline \multirow{2}{*}{ Edad } & \multicolumn{4}{|c|}{ Sufre estreñimiento } & \multirow{2}{*}{ Total } & \multirow{2}{*}{ P-value } \\
\hline & Nunca & Ocasional & Frecuente & Permanente & & \\
\hline$<20$ años & 4 & 4 & 0 & 0 & 8 & \multirow{8}{*}{0.000} \\
\hline de 20 a 29 años & 10 & 22 & 14 & 1 & 47 & \\
\hline de 30 a 39 años & 17 & 81 & 17 & 0 & 115 & \\
\hline de 40 a 49 años & 10 & 59 & 14 & 1 & 84 & \\
\hline de 50 a 59 años & 10 & 52 & 9 & 7 & 78 & \\
\hline de 60 a 69 años & 6 & 45 & 20 & 3 & 74 & \\
\hline$>69$ años & 2 & 0 & 0 & 3 & 5 & \\
\hline Total & 59 & 263 & 74 & 15 & 411 & \\
\hline
\end{tabular}

Los valores más significativos entre la edad y si sufre o no estreñimiento se observan en el grupo de los 30 años $(81,70.4 \%)$ debido, en parte, a la distribución que presenta la muestra. Los grupos de mayor propensión a sufrirlo permanentemente son los registrados entre los 
50 , los 60 y los mayores de 69 años $(13,72 \%)$ (7, 9\%; 3, 4.1\%; y 3, 60\%, respectivamente) considerándose como los más sensibles a la cronicidad de la afección.

Tabla 5. Tabla de contingencia Edad * Frecuencia de evacuación.

\begin{tabular}{|c|c|c|c|c|c|c|c|}
\hline \multirow[b]{2}{*}{ Edad } & \multicolumn{5}{|c|}{ Frecuencia de evacuación } & \multirow{2}{*}{ Total } & \multirow{2}{*}{$\begin{array}{c}\text { P- } \\
\text { value }\end{array}$} \\
\hline & $\begin{array}{c}1-2 \\
/ \mathrm{s}\end{array}$ & $\begin{array}{c}3-4 \\
/ s\end{array}$ & $\begin{array}{c}5-6 \\
/ s\end{array}$ & $\begin{array}{c}1-2 \\
/ d\end{array}$ & $\begin{array}{c}3 \text { o más } \\
/ d\end{array}$ & & \\
\hline$<20$ años & 0 & 2 & 0 & 6 & 0 & 8 & \multirow{8}{*}{0.006} \\
\hline de 20 a 29 años & 8 & 12 & 4 & 23 & 0 & 47 & \\
\hline de 30 a 39 años & 6 & 25 & 9 & 74 & 1 & 115 & \\
\hline de 40 a 49 años & 8 & 17 & 7 & 51 & 1 & 84 & \\
\hline de 50 a 59 años & 11 & 12 & 17 & 38 & 0 & 78 & \\
\hline de 60 a 69 años & 8 & 24 & 11 & 30 & 1 & 74 & \\
\hline$>69$ años & 3 & 0 & 0 & 2 & 0 & 5 & \\
\hline Total & 44 & 92 & 48 & 224 & 3 & 411 & \\
\hline
\end{tabular}

El grupo de los 50 es quien, posiblemente con motivo de la ralentización metabólica y el sedentarismo, presenta una irregularidad significativa en la frecuencia de evacuación (11, $14.1 \%$ ), a pesar de que en la distribución de la muestra se observa una mayor participación del grupo de los $30(6,5.2 \%)$.

Tabla 6. Tabla de contingencia Nivel de estudios * Sufre estreñimiento.

\begin{tabular}{|c|c|c|c|c|c|c|}
\hline \multirow{2}{*}{$\begin{array}{l}\text { Nivel de } \\
\text { estudios }\end{array}$} & \multicolumn{4}{|c|}{ Sufre estreñimiento } & \multirow{2}{*}{ Total } & \multirow{2}{*}{ P-value } \\
\hline & Nunca & Ocasional & Frecuente & Permanente & & \\
\hline Sin estudios & 1 & 11 & 4 & 5 & 21 & \multirow{6}{*}{0.000} \\
\hline Primaria & 12 & 66 & 11 & 1 & 90 & \\
\hline ESO & 8 & 39 & 16 & 5 & 68 & \\
\hline Bachiller & 8 & 55 & 12 & 0 & 75 & \\
\hline Universidad & 30 & 92 & 31 & 4 & 157 & \\
\hline Total & 59 & 263 & 74 & 15 & 411 & \\
\hline
\end{tabular}

El estreñimiento permanente (o crónico) es ligeramente más acusado en los grupos de menor nivel de estudios -sin estudios, primaria y ESO, (11, 73.3\%)- (5, 33.3\%, 1, 6.7\% y 5, $33.3 \%$, respectivamente), que en los grupos de mayor nivel formativo -universitarios- (4, $26.7 \%$ ). A mayor nivel cultural y de conocimiento, mayor es la concienciación acerca de los hábitos de vida saludables en general o de la condición del estreñimiento en particular. 
Tabla 7. Tabla de contingencia Nivel de estudios * Frecuencia de evacuación.

\begin{tabular}{|c|c|c|c|c|c|c|c|}
\hline \multirow{2}{*}{$\begin{array}{l}\text { Nivel de } \\
\text { estudios }\end{array}$} & \multicolumn{5}{|c|}{ Frecuencia de ir al baño } & \multirow[b]{2}{*}{ Total } & \multirow[b]{2}{*}{ P-value } \\
\hline & $\begin{array}{c}1-2 \\
/ s\end{array}$ & $\begin{array}{c}3-4 \\
/ s\end{array}$ & $\begin{array}{c}5-6 \\
/ s\end{array}$ & $\begin{array}{c}1-2 \\
/ d\end{array}$ & 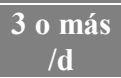 & & \\
\hline Sin estudios & 5 & 8 & 4 & 4 & 0 & 21 & \multirow{6}{*}{0.000} \\
\hline Primaria & 8 & 15 & 13 & 54 & 0 & 90 & \\
\hline ESO & 12 & 14 & 3 & 37 & 2 & 68 & \\
\hline Bachiller & 12 & 25 & 9 & 28 & 1 & 75 & \\
\hline Universitarios & 7 & 30 & 19 & 101 & 0 & 157 & \\
\hline Total & 44 & 92 & 48 & 224 & 3 & 411 & \\
\hline
\end{tabular}

En el nivel de Bachiller es donde se observan los resultados más diferenciados de la muestra $(37,49.3 \%)$ con frecuencias por debajo de las consideradas como el umbral del estreñimiento ( 3 veces a la semana). Como se puede apreciar es casi la mitad del porcentaje del conjunto de respuestas, lo que puede ser un reflejo de la percepción que los propios pacientes poseen de la sintomatología de la afección que según Longstreth, Thompson, \& Chey (2006) es muy habitual.

Tabla 8. Tabla de contingencia Sufre estreñimiento * Frecuencia de evacuación.

\begin{tabular}{|c|c|c|c|c|c|c|c|}
\hline \multirow{2}{*}{$\begin{array}{c}\text { Sufre } \\
\text { estreñimiento }\end{array}$} & \multicolumn{5}{|c|}{ Frecuencia de ir al baño } & \multirow[b]{2}{*}{ Total } & \multirow[b]{2}{*}{ P-value } \\
\hline & $\begin{array}{l}1- \\
2 / s\end{array}$ & $\begin{array}{c}3-4 \\
/ \mathrm{s}\end{array}$ & $\begin{array}{c}5-6 \\
/ s\end{array}$ & $\begin{array}{c}1-2 \\
/ d\end{array}$ & 3 o más /d & & \\
\hline No, nunca & 0 & 4 & 4 & 51 & 0 & 59 & \multirow{5}{*}{0.000} \\
\hline Ocasionalmente & 15 & 48 & 36 & 161 & 3 & 263 & \\
\hline Frecuentemente & 22 & 37 & 6 & 9 & 0 & 74 & \\
\hline Permanentemente & 7 & 3 & 2 & 3 & 0 & 15 & \\
\hline Total & 44 & 92 & 48 & 224 & 3 & 411 & \\
\hline
\end{tabular}

Un 64\% (263) del total de personas encuestadas (411) aseguran sufrir estreñimiento ocasionalmente. De los cuales un 5.7\% (15) presentan unos valores de frecuencia de evacuación habitual de 1 ó 2 veces semanales lo que podría estar causado por una percepción errónea de la frecuencia de evacuación, como aseguran algunas fuentes que es frecuente en algunos pacientes crónicos (American College of Gastroenterology, 2005).

A continuación se presentan las tablas resultantes de la aplicación del modelo ANOVA mediante la comparación de las medias. 
Estudio demoscópico de los atributos de diseño de un dispositivo de asistencia abdomino-intestinal.

Tabla 9. Método ANOVA y medias del factor Género.

\begin{tabular}{lrrrrrrrr}
\multicolumn{1}{c}{ Variables } & $\begin{array}{c}\text { Suma de } \\
\text { cuadrados }\end{array}$ & $\begin{array}{c}\text { Media } \\
\text { cuadrática }\end{array}$ & F & Sig. & $\begin{array}{c}\text { Media } \\
\text { hombr } \\
\text { e }\end{array}$ & $\begin{array}{c}\text { Media } \\
\text { Mujer }\end{array}$ & $\begin{array}{c}\text { Media } \\
\text { total }\end{array}$ \\
Desmontable a mano & 327,197 & 11,554 & 14,971 &, 000 & $(3,01)$ & $(3,34)$ & $(3,19)$ \\
Plegable & 383,387 & 16,982 & 18,957 &, 000 & $(2,95)$ & $(3,36)$ & $(3,18)$ \\
Apariencia compleja & 474,428 & 15,553 & 13,863 &, 000 & $(2,41)$ & $(2,02)$ & $(2,19)$ \\
\hline
\end{tabular}

El valor de las medias obtenidas denota que el sector femenino muestra un mayor grado de acuerdo en la consideración de los atributos de diseño, como: Desmontable a mano (3.34), y plegable (3.36), mientras que el sector masculino destaca en Apariencia compleja (2.41).

Tabla 10. Método ANOVA y medias del factor Edad.

\begin{tabular}{|c|c|c|c|c|c|c|c|c|c|}
\hline \multirow{3}{*}{ Variables } & \multicolumn{7}{|c|}{ Edad } & \multirow{3}{*}{$\begin{array}{l}\text { Total } \\
\text { Media }\end{array}$} & \multirow{3}{*}{ Sig. } \\
\hline & $<20$ & $20-29$ & $30-39$ & $40-49$ & $50-59$ & $60-69$ & $>69$ & & \\
\hline & Media & Media & Media & Media & Media & Media & Media & & \\
\hline Ecológico & $(2,00)$ & $(3,47)$ & $(3,34)$ & $(3,07)$ & $(3,17)$ & $(3,12)$ & $(2,80)$ & $(3,19)$ & ,003 \\
\hline Personalizable & $(2,88)$ & $(2,68)$ & $(2,56)$ & $(2,58)$ & $(2,13)$ & $(2,74)$ & $(1,00)$ & $(2,52)$ &, 000 \\
\hline Especializado & $(2,25)$ & $(2,53)$ & $(2,57)$ & $(2,49)$ & $(2,28)$ & $(2,77)$ & $(1,40)$ & $(2,51)$ &, 001 \\
\hline Versátil & $(3,25)$ & $(2,47)$ & $(2,80)$ & $(2,96)$ & $(2,94)$ & $(3,11)$ & $(4,00)$ & $(2,90)$ &, 000 \\
\hline Robusto & $(3,50)$ & $(2,55)$ & $(2,91)$ & $(3,12)$ & $(3,21)$ & $(2,82)$ & $(3,60)$ & $(2,97)$ & ,001 \\
\hline $\begin{array}{l}\text { Respaldo } \\
\text { científico }\end{array}$ & $(2,13)$ & $(3,45)$ & $(3,41)$ & $(3,45)$ & $(3,44)$ & $(3,35)$ & $(4,00)$ & $(3,40)$ & ,001 \\
\hline $\begin{array}{l}\text { Apariencia } \\
\text { gimnasio }\end{array}$ & $(2,25)$ & $(1,70)$ & $(2,17)$ & $(2,39)$ & $(2,29)$ & $(2,66)$ & $(2,40)$ & $(2,28)$ &, 000 \\
\hline $\begin{array}{l}\text { Apariencia } \\
\text { máquina } \\
\text { doméstica }\end{array}$ & $(2,38)$ & $(2,09)$ & $(2,06)$ & $(2,45)$ & $(2,60)$ & $(2,32)$ & $(2,60)$ & $(2,31)$ &, 004 \\
\hline $\begin{array}{l}\text { Estética } \\
\text { innovadora }\end{array}$ & $(3,13)$ & $(2,85)$ & $(2,64)$ & $(3,11)$ & $(3,12)$ & $(3,20)$ & $(3,20)$ & $(2,97)$ &, 001 \\
\hline $\begin{array}{l}\text { Integrado en } \\
\text { baño }\end{array}$ & $(3,38)$ & $(3,30)$ & $(3,17)$ & $(3,57)$ & $(3,45)$ & $(3,42)$ & $(2,40)$ & $(3,36)$ &, 005 \\
\hline $\begin{array}{l}\text { Poseer motivos } \\
\text { decorativos }\end{array}$ & $(2,50)$ & $(2,09)$ & $(1,77)$ & $(2,02)$ & $(1,88)$ & $(1,78)$ & $(1,00)$ & $(1,89)$ &, 002 \\
\hline $\begin{array}{l}\text { Supervisado por } \\
\text { especialista }\end{array}$ & $(1,88)$ & $(2,26)$ & $(2,87)$ & $(2,54)$ & $(2,73)$ & $(2,51)$ & $(2,80)$ & $(2,62)$ & ,002 \\
\hline Usado en centro & $(2,25)$ & $(2,81)$ & $(2,54)$ & $(2,89)$ & $(2,51)$ & $(2,85)$ & $(1,00)$ & $(2,67)$ &, 000 \\
\hline $\begin{array}{l}\text { Accionamiento } \\
\text { manual }\end{array}$ & $(2,25)$ & $(2,87)$ & $(2,97)$ & $(3,21)$ & $(3,37)$ & $(3,51)$ & $(3,40)$ & $(3,17)$ &, 001 \\
\hline
\end{tabular}

Cruzando las variables con el factor Edad, se puede observar como el grupo de menores de 20 años y los de entre 40 y 49 años, muestran valoraciones semejantes a nivel general, exceptuando el uso en centro sanitario y que posea apariencia de máquina de gimnasio, peor valoradas por los menores de 20 . No obstante, este último grupo presenta medias muy superiores en sus valoraciones, destacando la cualidad de que sea robusto (3.50) y que 
posea motivos decorativos (2.50). Entre los grupos de los 20 y los 30, destacan similitudes sobre que sea ecológico (3.47 y 3.34), personalizable (2.68 y 2.56) y especializado (2.53) y 2.57), no así, en poseer motivos decorativos (2.09) y usado en centro sanitario (2.81), mejor valoradas por el grupo de los 20 años; y ser supervisado por un especialista (2.87) y necesitar consumo eléctrico (1.63) mejor valorados por los de 30 años. Los grupos de los de 40 y 60 años, muestran el mayor número de valoraciones por encima de la media, sin embargo, junto con los de 50 años, se destaca la elevada heterogeneidad en las mismas. Esto es debido, quizás, a que siendo los más exigentes, poseen diferentes criterios por la disparidad de sus entornos, lo que afecta a sus condiciones de vida, personalidades y, en consecuencia, a sus prioridades.

Tabla 11. Método ANOVA y medias del factor Nivel de estudios.

\begin{tabular}{|c|c|c|c|c|c|c|c|}
\hline \multirow[b]{2}{*}{ Variables } & \multicolumn{5}{|c|}{ Nivel de estudios } & \multirow[b]{2}{*}{$\begin{array}{l}\text { Total } \\
\text { Media }\end{array}$} & \multirow[b]{2}{*}{ Sig. } \\
\hline & $\begin{array}{c}\text { Sin } \\
\text { estudios } \\
\text { Media }\end{array}$ & $\begin{array}{c}\text { Primaria } \\
\text { Media }\end{array}$ & $\begin{array}{c}\text { ESO } \\
\text { Media }\end{array}$ & $\begin{array}{c}\text { Bachiller } \\
\text { Media }\end{array}$ & $\begin{array}{c}\text { Universitarios } \\
\text { Media }\end{array}$ & & \\
\hline Portátil & $(2,86)$ & $(3,44)$ & $(3,38)$ & $(3,17)$ & $(2,98)$ & $(3,18)$ & ,002, \\
\hline Desmontable a mano & $(3,05)$ & $(3,39)$ & $(3,37)$ & $(3,23)$ & $(3,00)$ & $(3,19)$ &, 005 \\
\hline Versátil & $(3,19)$ & $(3,18)$ & $(2,96)$ & $(2,92)$ & $(2,67)$ & $(2,90)$ &, 000 \\
\hline Apariencia gimnasio & $(2,62)$ & $(2,58)$ & $(2,40)$ & $(2,32)$ & $(1,99)$ & $(2,28)$ &, 000 \\
\hline $\begin{array}{l}\text { Apariencia máquina } \\
\text { doméstica }\end{array}$ & $(2,62)$ & $(2,43)$ & $(2,54)$ & $(2,37)$ & $(2,06)$ & $(2,31)$ & ,001 \\
\hline Estética atrevida & $(2,86)$ & $(1,83)$ & $(2,12)$ & $(2,27)$ & $(2,27)$ & $(2,18)$ &, 000 \\
\hline Estética innovadora & $(3,14)$ & $(3,18)$ & $(3,19)$ & $(2,93)$ & $(2,75)$ & $(2,97)$ & 001 \\
\hline Integrado en baño & $(3,10)$ & $(3,64)$ & $(3,35)$ & $(3,32)$ & $(3,25)$ & $(3,36)$ &, 005 \\
\hline Apariencia compleja & $(2,90)$ & $(2,08)$ & $(2,22)$ & $(2,49)$ & $(2,01)$ & $(2,19)$ &, 000 \\
\hline Usado en centro & $(2,71)$ & $(2,96)$ & $(2,94)$ & $(2,72)$ & $(2,36)$ & $(2,67)$ &, 000 \\
\hline Herramientas montaje & $(1,76)$ & $(1,90)$ & $(1,96)$ & $(1,49)$ & $(1,74)$ & $(1,77)$ & ,003 \\
\hline Necesitar instalación & $(2,19)$ & $(2,33)$ & $(2,24)$ & $(1,99)$ & $(1,76)$ & $(2,03)$ &, 000 \\
\hline Accionamiento manual & $(3,19)$ & $(3,52)$ & $(3,35)$ & $(3,17)$ & $(2,89)$ & $(3,17)$ &, 000 \\
\hline
\end{tabular}

El grupo que muestra las valoraciones más elevadas es el grupo sin estudios, destacando: poseer estética atrevida (2.86) y apariencia compleja (2.90). Sin embargo, muestra mayor rechazo a ser portátil (2.86). Por el contrario, se observa como el grupo de formación universitaria penaliza que sea versátil (2.67), usado en centro sanitario (2.36), que necesite instalación (1.76) y accionamiento manual (2.89). 
Tabla 13. Método ANOVA y medias del factor Sufre estreñimiento.

\begin{tabular}{|c|c|c|c|c|c|c|}
\hline \multirow[b]{2}{*}{ Variables } & \multicolumn{4}{|c|}{ Sufre estreñimiento } & \multirow{2}{*}{$\begin{array}{l}\text { Total } \\
\text { Media }\end{array}$} & \multirow[b]{2}{*}{ Sig. } \\
\hline & $\begin{array}{l}\text { Nunca } \\
\text { Media }\end{array}$ & $\begin{array}{c}\text { Ocasional } \\
\text { Media }\end{array}$ & $\begin{array}{c}\text { Frecuente } \\
\text { Media }\end{array}$ & $\begin{array}{c}\text { Permanente } \\
\text { Media }\end{array}$ & & \\
\hline Personalizable & $(2,63)$ & $(2,38)$ & $(2,92)$ & $(2,40)$ & $(2,52)$ & ,001 \\
\hline Innovador & $(3,00)$ & $(3,35)$ & $(3,14)$ & $(3,07)$ & $(3,25)$ & ,002 \\
\hline Reciclable & $(3,34)$ & $(2,84)$ & $(3,14)$ & $(3,13)$ & $(2,98)$ &, 000 \\
\hline Robusto & $(3,00)$ & $(3,05)$ & $(2,61)$ & $(3,33)$ & $(2,97)$ &, 003 \\
\hline Estética atrevida & $(2,27)$ & $(2,06)$ & $(2,36)$ & $(3,07)$ & $(2,18)$ &, 000 \\
\hline Apariencia compleja & $(2,37)$ & $(1,98)$ & $(2,73)$ & $(2,60)$ & $(2,19)$ &, 000 \\
\hline Necesitar aprendizaje & $(1,93)$ & $(2,02)$ & $(1,57)$ & $(1,93)$ & $(1,92)$ &, 003 \\
\hline
\end{tabular}

En los grupos que nunca sufren estreñimiento o lo hacen ocasionalmente, no se muestran datos con un elevado nivel de significación, destacando en el primer grupo las valoraciones más bajas para innovador (3.00); o reciclable (2.84) y que posea una apariencia compleja (1.98) en el segundo. Se destaca, sin embargo, los grupos que sufren estreñimiento frecuentemente, y especialmente, de manera permanente, los que muestran mayores niveles de valoración, en: que posea apariencia compleja (2.73 y 2.60, respectivamente).

Tabla 15. Comparación de medias del factor Frecuencia de evacuación.

\begin{tabular}{|c|c|c|c|c|c|c|c|}
\hline \multirow[b]{2}{*}{ Variables } & \multicolumn{5}{|c|}{ Frecuencia de ir al baño } & \multirow[b]{2}{*}{$\begin{array}{l}\text { Total } \\
\text { Media }\end{array}$} & \multirow[b]{2}{*}{ Sig. } \\
\hline & $\begin{array}{c}1-2 \\
/ \mathrm{s} \\
\text { Media }\end{array}$ & $\begin{array}{c}3-4 \\
/ \mathrm{s} \\
\text { Media }\end{array}$ & $\begin{array}{c}5-6 \\
/ \mathrm{s} \\
\text { Media }\end{array}$ & $\begin{array}{l}\text { 1- } 2 / d \\
\text { Media }\end{array}$ & $\begin{array}{c}3 \text { o más } \\
\text { /d } \\
\text { Media }\end{array}$ & & \\
\hline Personalizable & $(2,68)$ & $(2,88)$ & $(2,63)$ & $(2,31)$ & $(2,67)$ & $(2,52)$ & 000 \\
\hline Sofisticado & $(2,66)$ & $(2,79)$ & $(2,21)$ & $(2,14)$ & $(3,67)$ & $(2,36)$ &, 000 \\
\hline Innovador & $(3,09)$ & $(3,11)$ & $(2,96)$ & $(3,40)$ & $(3,33)$ & $(3,25)$ &, 000 \\
\hline Portátil & $(3,23)$ & $(2,83)$ & $(3,08)$ & $(3,33)$ & $(3,67)$ & $(3,18)$ &, 002 \\
\hline Duradero & $(3,23)$ & $(3,23)$ & $(3,33)$ & $(3,58)$ & $(4,00)$ & $(3,44)$ &, 002 \\
\hline Desechable & $(2,32)$ & $(2,50)$ & $(1,96)$ & $(2,12)$ & $(3,33)$ & $(2,21)$ &, 001 \\
\hline Plegable & $(3,27)$ & $(2,92)$ & $(2,77)$ & $(3,35)$ & $(3,00)$ & $(3,18)$ &, 000 \\
\hline Adaptable & $(3,30)$ & $(3,15)$ & $(3,19)$ & $(3,51)$ & $(3,67)$ & $(3,37)$ &, 002 \\
\hline Ergonómico & $(3,45)$ & $(3,30)$ & $(3,15)$ & $(3,59)$ & $(4,00)$ & $(3,46)$ &, 000 \\
\hline Integrado en salón & $(2,20)$ & $(1,82)$ & $(1,73)$ & $(1,60)$ & $(2,67)$ & $(1,74)$ & 001 \\
\hline Apariencia limpia & $(3,00)$ & $(3,12)$ & $(3,21)$ & $(3,45)$ & $(4,00)$ & $(3,30)$ & 002 \\
\hline Apariencia compleja & $(2,98)$ & $(2,87)$ & $(2,02)$ & $(1,79)$ & $(3,00)$ & $(2,19)$ &, 000 \\
\hline Necesitar aprendizaje & $(1,77)$ & $(1,65)$ & $(1,88)$ & $(2,06)$ & $(2,33)$ & $(1,92)$ &, 004 \\
\hline $\begin{array}{l}\text { Necesitar herramientas } \\
\text { para montaje }\end{array}$ & $(1,70)$ & $(1,52)$ & $(1,67)$ & $(1,89)$ & $(2,33)$ & $(1,77)$ & 001 \\
\hline
\end{tabular}

La comparación de las medias obtenidas arroja, irónicamente, una mayor valoración en los grupos de mayor frecuencia de evacuación, destacando al grupo de personas que evacua 
más de tres veces al día como los que mayores puntuaciones poseen. Una posible explicación a este hecho podría ser que sufren la condición opuesta $\mathrm{y}$, esto da lugar, a un reflejo social solidario.

Los grupos de menor frecuencia (1 ó 2 y 3 ó 4 veces a la semana) muestran mayores puntuaciones en el grado de sofisticación (2.66 y 2.79), integrado en el salón (2.20, en el primer grupo) y que posea apariencia compleja (2.98 y 2.87 , respectivamente). Sin embargo, estos grupos muestran los valores mínimos en cuanto a que posea apariencia limpia (3.00), que necesite aprendizaje de uso (1.65) y que requiera de herramientas de montaje (1.52). Esto puede deberse a la consideración de la afección como una condición sanitaria compleja y, por lo tanto, el dispositivo debe pertenecer a la tipología de producto de uso clínico, en los que existe un elevado nivel tecnológico.

\section{Conclusión}

A la vista de los resultados obtenidos se puede decir que en la muestra predomina el género femenino, en edades entre la treintena y la madurez, con alto nivel de formación, que sufre estreñimiento ocasionalmente y posee unos hábitos defecatorios dentro de la normalidad. Se puede considerar que la muestra es significativa y se encuentra dentro de los valores presentados en los informes de instituciones de carácter oficial como el Instituto Nacional de Estadística (INE, 2016).

Los resultados de los análisis estadísticos sobre la opinión de los posibles usuarios mostraron, en primer lugar, la importancia de algunas variables -como la edad y el géneroque influyen directamente sobre la afección, diferenciando grupos poblacionales con mayor índice de prevalencia del estreñimiento. Tal es el caso del sector femenino, de mediana edad, con apenas estudios, que junto con las personas mayores, son quienes presentan el mayor índice de estreñimiento, corroborando de esta manera las fuentes oficiales consideradas en la investigación (AEG, 2011). El nivel de estudios aparece como una variable de interés, ya que se encuentra presente como un factor a considerar en muchos de los casos. La interpretación que se baraja es que un mayor nivel cultural y formativo, puede reflejar una mayor concienciación sobre el estilo de vida saludable y los conocimientos o el interés por llevarlo a cabo.

En segundo lugar, los resultados de las valoraciones de los atributos propuestos para el diseño del dispositivo, mostraron una tendencia hacia la múltiple diferenciación de la tipología del producto, en función de sus perfiles. En base a lo expuesto, se identifican varios grupos poblacionales con intereses muy dispares, como son: las mujeres de mediana edad, quienes valoran por encima de todo la practicidad del dispositivo destacando, por encima del resto, atributos como: Desmontable a mano, y plegable. En oposición, se puede 
observar como la personalización, la sofisticación, la necesidad de aprendizaje de uso y el consumo eléctrico o de baterías, son las peor valoradas por dicho grupo, destacando entre todas ellas poseer apariencia compleja.

El género masculino en general destaca como atributos positivos: ser duradero, con respaldo científico y ser adaptable. Sin embargo las valoraciones de dichas características se encuentran por debajo de la media y no constituyen una aportación significativa. No obstante los hombres de mediana edad, destacan su preocupación por el consumo energético y necesitar aprendizaje de uso, pero valoran positivamente, la diferenciación, sofisticación y el grado de innovación. Una posible interpretación de los datos es que la mujer centra más su atención en características con notaciones relativas a la manipulación, la funcionalidad, la usabilidad y el pragmatismo. Cuestiones que podrían ser consecuencia de una preocupación por el ahorro de espacio y tiempo. Mientras que los hombres muestran mayor predisposición por el ahorro de energía.

Las personas mayores de 60 años muestran una preocupación por el uso supervisado por los especialistas y en centros sanitarios; y los menores de 30 años, quienes comparten su descontento con el uso en centros especializados, valoran positivamente que sea desechable, ecológico, personalizable y robusto.

Es interesante resaltar la distribución en los grupos de madurez donde los valores presentan una relevancia significativa frente a los grupos de menor edad, tal y como constatan las fuentes bibliográficas. Una posible explicación es el aumento del sedentarismo y la ralentización del metabolismo como sostiene Amir (2011), por el padecimiento de alguna otra afección relacionada que lo cause como mantiene Folden (2002) o la ingesta de algún fármaco que pueda influir como sostienen Harari, Gurwitz, \& Minaker (1993).

Por otro lado, la escasa participación del grupo mayores de 69 años puede deberse a que la encuesta fue realizada mayoritariamente de forma telemática $(293,71.2 \%)$ y por lo tanto, este rango de edad ha podido verse afectado.

\section{Referencias}

AEG. (2011). Tratamiento de las enfremedades gastroenterológicas. Madrid: Asociación Española de Gastroenterología.

American College of Gastroenterology. (2005). An evidence-based approach to the management of chronic constipation in North America. The American Jpurnal of Gastroenterology, S1-S4.

Amir, A. (2011). Etiological factors of constipation in the elderly, with enphasis on functional causes. Eastern Mediterranean Health Journal, 708-711. 
Arriaza, M. (2005). Guía práctica de análisis de datos. Sevilla: Consejería de innovación, ciencia y empresa.

Esnal, I., \& Serrano, C. (2013). Diseño y desarrollo de un prototipo para contribuir a la disminución del estreñimiento y patologías asociadas. Teisina de Máster. Valencia: Universitat Politècnica de València.

Folden, S. L. (2002). Practice guidelines for the management of constipation in adults. Glenview: Rehabilitation Nursing Fundation.

Harari, D., Gurwitz, J. H., \& Minaker, K. L. (1993). Constipation in the elderly. Journal of the American Geriatrics Society, 1130-1140.

INE. (2016). Cifras de población. Estadísticas de migraciones. Instituto Nacional de Estadística.

Longstreth, G. F., Thompson, W. G., \& Chey, W. D. (2006). Functional Bowel Disorders. Gastroenterology, 1480-1492.

López García, D. (2013). Analysis of the possibilities of use of Big Data in organizations. Santander: Universidad de Cantabria.

Malhorta, N. k. (2004). Investigación de mercados. México DF: Perason Educación.

Norman, D. A. (1988). The psychology of everyday things. New York: Basic Books.

Rome Fundation. (2006). The functional gastrointestinal disorders. Raleigh: Rome Fundation.

WGO. (2010). Constipation: A world perspective. En W. G. Organisation, Guias mundiales. Milwaukee: World Gastroenterology Organisation. 